\title{
Advanced Sensors Placement for Accurate 3D Needle Shape Reconstruction
}

\author{
Pierre-Loup Schaefer ${ }^{1}$, Grégory Chagnon ${ }^{1}$ and Alexandre Moreau-Gaudry ${ }^{1}$
}

\begin{abstract}
Needles are tools widely used in minimally invasive surgery. During such procedure the localization of the needle and its tip is a challenging situation because of the needle deformations due to the interactions with tissues. To tackle this problem, instrumented needles with sensors are currently developed to allow needle reconstruction and tip localization. In conventional surgery this difficulty is overcome by medical imaging. The interest of using an instrumented needle resides in the possible dispense of medical imaging. This papers develops new methods to reconstruct needles in three dimensions and to find the locations of sensors which minimizes the error of reconstruction of the needle. A notable feature of our method is that input data are based on real needle data, that should assure a better representativity of our results. Reconstructions simulated with 22 gauges $200 \mathrm{~mm}$ long needles data show that the localization of the needle tip is more accurate by $18 \%$ to $52 \%$ with optimal sensors positions compared to equidistant sensors positions.
\end{abstract}

\section{INTRODUCTION}

Needles are more and more used in percutaneous medical procedures, such as, for instance, during interventional Radiology Procedures. During these procedures, the knowledge of the needle location is of primary importance with stakes being no others than the success or the failure of the procedures. Nevertheless, accurate location of the needle may be challenging because of the bending phenomenon which might appear when the needle is inserted. This phenomenon is caused by interactions between needle and tissue. It is also influenced by a lot of factors such as the size of the needle, its length or the type of tissues. Insertions in phantom using 18 gauges $20 \mathrm{~cm}$ have shown that tip deflection can be up to $2.8 \mathrm{~mm}$ for a $6 \mathrm{~cm}$ insertion [16] and up to $12 \mathrm{~mm}$ for a $10 \mathrm{~cm}$ insertion [12]. Furthermore deflections of 22 gauges $20 \mathrm{~cm}$ needles used for insertions in pig tissues can be as high as $25 \mathrm{~mm}$ [14]. As a matter of fact, while using needles during minimally-invasive medical procedures, with no assisting tools, incertainty on the position remains. Furthermore current computer assisted interventional radiology systems make the assumption of non-deformability of the needle [3], [9] and then don't provide direct access to the real shape of the needle, still needing additional medical imaging.

This article presents a method to reconstruct the 3D shape of a needle from the data retrieved from its sensors and a procedure to find the best locations of the sensors to minimize the tip localization error. The paper is organized as follows: Section II explains the three-dimensional reconstruction of the needle shape from sensors data. Section III describes the

\footnotetext{
*This work was supported by the French ANR GAME-D

${ }^{1}$ Univ. Grenoble Alpes / CNRS / TIMC-IMAG UMR 5525, France. Email: FirstName. Lastnamedimag.fr
}

method for evaluating best sensors position by processing real needle data. Section IV present and analyze the results, and is followed by Section V on future improvements.

\section{3D NeEdle Shape Reconstruction}

\section{A. Sensors informations}

This section presents the process to obtain geometrical informations of the needle from the sensors. The purpose of the sensors is to measure the strain applied on the needle during the insertion. Differents types of sensors include optical fiber Bragg gratings [1], [11] and strain gauges [18], [19]. From a sensor triplet it is possible to obtain two geometrical parameters at the sensor location: the curvature $\kappa$ and the bending angle $\theta$ [1], [15]. Let $\left(s_{i}\right)_{i=1, . ., n}$ the value such as $\left(\mathbf{M}\left(s_{i}\right)\right)_{i=1, . ., n}$ are points of the needle where the triplets are located. Then for the triplet located at $s_{i}$ we can retrieve the curvature $\kappa_{i}=\kappa\left(s_{i}\right)$ and the bending angle $\theta_{i}=\theta\left(s_{i}\right)$. The set $\left(\kappa\left(s_{i}\right)\right)_{i=1, \ldots, n}$ and $\left(\theta\left(s_{i}\right)\right)_{i=1, \ldots, n}$ constitutes a discretization of the function $\kappa$ and $\theta$. The interpolations of both sets gives us $\kappa_{e s t}$ and $\theta_{\text {est }}$, the estimate of curvature and bending angle from the sensors data.

\section{B. Shape reconstruction}

This section presents the method to obtain 3D needle shape estimation from the curvature and bending angle estimate $\kappa_{e s t}$ and $\theta_{\text {est }}$. Due to the geometry of the needles and the mechanical interactions with the tissues we make the widely accepted hypothesis that the mechanical torsion of needles is negligible [1], [11], [20]. Given this assumption our reconstruction will be based on Rotation Minimizing Frame. Rotation Minimizing Frame (RMF) belongs to the family of 3D moving frame. A 3D moving frame is a frame composed of an orthonormal ordered basis vector defined along a space curve. In the rest of the study we use the notation $\left(\mathbf{T}, \mathbf{N}_{\mathbf{1}}, \mathbf{N}_{\mathbf{2}}\right)$ to refer to the RMF. We have $\mathbf{T}, \mathbf{N}_{\mathbf{1}}, \mathbf{N}_{\mathbf{2}} \in \mathbb{R}^{3}$ and $\mathbf{T}$ is tangent to the curve. Differents moving frames can be defined for the same curve [2] and RMF has the property of minimizing its twisting motion when framing a curve [17]. Combined with the negligible torsion hypothesis this property results in the RMF being a materially fixed basis of the beam [5]. Calculus of the Cartan matrix under such assumptions give the following relationship:

$$
\frac{d}{d s}\left[\begin{array}{c}
\mathbf{T}(s) \\
\mathbf{N}_{\mathbf{1}}(s) \\
\mathbf{N}_{\mathbf{2}}(s)
\end{array}\right]=\kappa(s)\left[\begin{array}{ccc}
0 & \cos \theta(s) & -\sin \theta(s) \\
-\cos \theta(s) & 0 & 0 \\
\sin \theta(s) & 0 & 0
\end{array}\right]\left[\begin{array}{c}
\mathbf{T}(s) \\
\mathbf{N}_{\mathbf{1}}(s) \\
\mathbf{N}_{\mathbf{2}}(s)
\end{array}\right]
$$

The signification of Eq. 1 is that for every point of the needle $\mathbf{M}(s)$ with $s \in[0, L]$, where $L$ is the length of the 
needle, there is a relation at this point between the material frame $\left(\mathbf{T}(s), \mathbf{N}_{\mathbf{1}}(s), \mathbf{N}_{\mathbf{2}}(s)\right)$ and its derivative involving $\kappa(s)$ and $\theta(s)$. Assuming known $\kappa_{e s t}$ and $\theta_{e s t}$, the estimate of curvature and bending angle from the sensors, and under appropriate boundaries conditions, the resolution of differential equation system Eq.1 gives the estimate of the RMF of the needle $\left(\mathbf{T}_{\text {est }}, \mathbf{N} \mathbf{1}_{\text {est }}, \mathbf{N} \mathbf{2}_{\text {est }}\right)$. Finally the estimate of the needle shape $\mathbf{M}_{\text {est }}$ is obtained by integrating $\mathbf{T}_{\text {est }}$ :

$$
\mathbf{M}_{\mathbf{e s t}}(s)=\mathbf{M}_{\mathbf{0}}+\int_{s_{0}}^{s} \mathbf{T}_{\mathbf{e s t}}(x) d x, \quad s \in[0, L]
$$

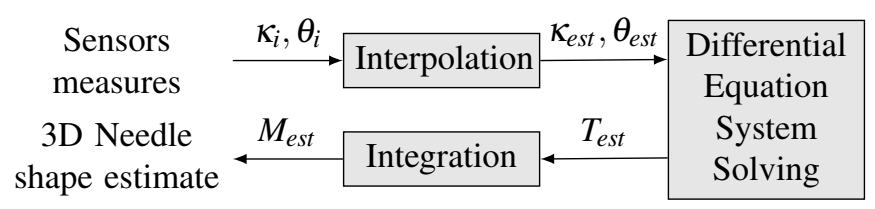

Fig. 1. Flowchart indicating the steps of the 3D Needle Shape Reconstruction using the sensors measures.

\section{SENSORS PLACEMENT}

\section{A. Method}

Sensor placement is a relatively few documented subject with [11] and [14] being the main articles on the subject. Approches made in both articles are to consider the optimal sensor location problem as a minimization one. The optimal sensor location is then defined as the location which minimizes the error made when reconstructing shapes with sensors at a certain location. Process used for evaluating the error of reconstruction on a needle for a given sensor location has the following steps. Firstly is defined a set of reference needles as input data. In both cases, these needles are calculated with 2D beam theory using force loads. Loads used by Park and al. [11] are constitued of equidistantly reparted 2D forces over the needle with arbitrary amplitudes, while loads used by Robert and al. [14] are more representative as the $2 \times 2 \mathrm{D}$ forces comes from a preliminary study conducted on real needles. Then a calculation is made for given sensors locations, representing what the sensors output would be if they were really present at that place on each of the needle of the reference set. Under the $2 \mathrm{D}$ assumption the output of the sensors take the form of simple curvature. Eventually these values are used to reconstruct a needle shape which is then compared to the reference needle. Quality measurement of the reconstruction is made by using the tip deflection error, a widely used medical criteria for needle precision when performing a surgical act.

The method of sensor location optimization presented in this article differs from these previous methods by the use of a reference set based on real needle data and by the three-dimensional procedures, resulting in a more exhaustive approach. Fig. 2 presents an overview of the differents steps of our method.

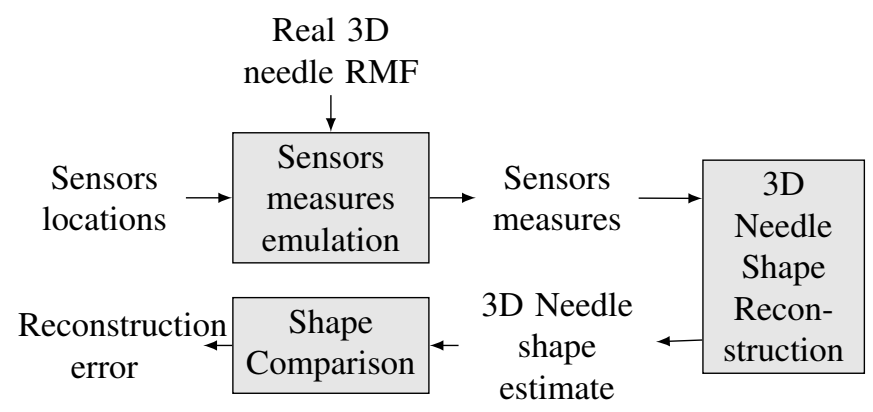

Fig. 2. Flowchart illustrating the evaluation of the reconstruction error from sensors location over a set of real needles

\section{B. Study of a set of needle}

The aim of this section is to generate the reference set of needles which will be used in sensor location optimization process. We retrieve the needle shape from real needle data by B-Spline smoothing and then we compute the RMF of the B-Spline. The RMF will be used to simulate the data of the sensors (Fig.2: Real 3D needle RMF).

1) Data: The study was carried out on a set of $54 \mathrm{CT}$ scans of needle insertion into pig shoulder [14]. Experiments involved standart $200 \mathrm{~mm}$ long stainless steel 22 gauges needles, which are often used in interventional radiology procedures. Choice of pig tissues is due to its similar biomechanical properties with human tissues [7].

2) Segmentation: In order to retrieve the voxels representing the needle we performed a segmentation on each of the scan. The aim is to separate the needle from the surrounding tissues, bones, etc... A region growing algorithm is particularly indicated in that case because of the difference of density of the needle and the density of the organs and tissues. We implemented the algorithm in a plug-in of the medical visualisation software CamiTK [6]. Once the segmentation is processed stays only the voxels of the needle. We then have 54 data sets of voxels.

3) Smoothing: The main objective of this part is to reconstruct the shape of the needle from its voxels. The mathematical representation of the shape is the three-dimensional univariate mathematical function which maps the length between the proximal extremity and one point of the needle to the three-dimensional coordinates of this point. Needle voxels are the results of a noisy spatial discretization of that function, consequently it is possible to approximate the shape function by performing smoothing on needle voxels [13]. The smoothing used here will be B-Spline smoothing, one of the most commonly used methods to smooth data. It consists to perform a regularized regression on a B-Spline basis. 3D BSplines are piecewise polynomials defined by their order $n$, their knots $\left(t_{i}\right)_{i=1, \ldots, m}$ and their control points $\left(P_{i}\right)_{i=1, \ldots, n_{c}} \in \mathbb{R}^{3}$ [4]. Let $S(t)$ be the B-Spline which is defined by:

$$
S(t)=\sum_{i=1}^{n_{c}} B_{i, n}(t) P_{i}
$$


with the following recursive definition:

$$
\begin{gathered}
B_{i, 0}(t)= \begin{cases}1 & \text { if } t_{i} \leq t \leq t_{i+1} \\
0 & \text { otherwise }\end{cases} \\
B_{i, n}(t)=\frac{t-t_{i}}{t_{i+n}-t_{i}} B_{i, n-1}(t)+\frac{t_{i+n+1}-t}{t_{i+n+1}-t_{i+1}} B_{i+1, n-1}(t)
\end{gathered}
$$

Let the data points $\left(Y_{i}\right)_{i=1, . ., n_{d}}$ be the voxels coordinates and $\left(w_{i}\right)_{i=1, \ldots, n_{d}}$ be their values. Let $\left(\bar{t}_{i}\right)_{i=1, . ., n_{d}}$ be the parameterization associed with the data points. The smoothing system then takes the following form:

$$
\sum_{l=1}^{n_{d}} w_{l}\left\|S\left(\bar{t}_{l}\right)-Y_{l}\right\|^{2}+\lambda \int_{0}^{1}\left\|S^{\prime \prime}(t)\right\|^{2} d t
$$

The smoothing B-Spline estimate is defined to be the minimizer of the Eq. 6 over the control points $\left(P_{i}\right)_{i=1, \ldots, n_{c}}$. The left part of Eq. 6 reflects accuracy to the data points while the right part express the roughness of the estimate. The trade-off between these two parts is the variable $\lambda$, called the smoothing parameter. Because the problem of smoothing noisy data with a spline is well posed, the system admits one and only one solution. Thus, as a result, we have a set of 54 3D B-Spline.

4) Rotation Minimizing Frame: Each B-Spline estimate is then processed to determine its RMF. Method used to compute the RMF is called Double reflection method [17]. Choice of this method in particular is justified by its stability and its global approximation error of high order. Finally we obtain a set of $54 \mathrm{RMF}$ corresponding to the material frames of the needles scanned in the input data.

\section{Minimization}

Finding optimal sensors positions is a $n$-dimensional problem, with $n$ being the number of sensors. It belongs to the Mixed Integer Nonlinear Programming (MINLP) family as it is a problem with a nonlinear objective function (the reconstruction error) and that we restrain the sensors positions to integer (precision on the positions of the sensors doesn't need to be submilimeter). As the convexity of our problem is undetermined we used global optimization solvers (Genetic Algorithm [10] and NOMAD [8]).

\section{Results}

This section presents results from our study on optimal sensor locations on 22 gauges $200 \mathrm{~mm}$ long needles set.

\begin{tabular}{|c|c|c|c|c|c|c|c|}
\hline \multirow{2}{*}{ Number of Sensors } & \multicolumn{7}{|c|}{ Sensors locations (mm) } \\
\cline { 2 - 8 } & 1 & 2 & 3 & 4 & 5 & 6 & 7 \\
\hline 1 & 81 & & & & & & \\
\hline 2 & 25 & 99 & & & & & \\
\hline 3 & 23 & 74 & 139 & & & & \\
\hline 4 & 18 & 57 & 103 & 150 & & & \\
\hline 5 & 16 & 40 & 73 & 118 & 157 & & \\
\hline 6 & 17 & 37 & 67 & 107 & 146 & 174 & \\
\hline 7 & 17 & 37 & 67 & 104 & 128 & 150 & 174 \\
\hline
\end{tabular}

TABLE I

TIP DEFLECTION ERROR DEPENDING TO THE NUMBER OF SENSORS
Table I presents the result of the minimization problem presented in Section III. It shows the optimal location of the sensors for a number of sensor going from one to seven. The optimization solvers didn't converge for a number of sensors superior to seven.

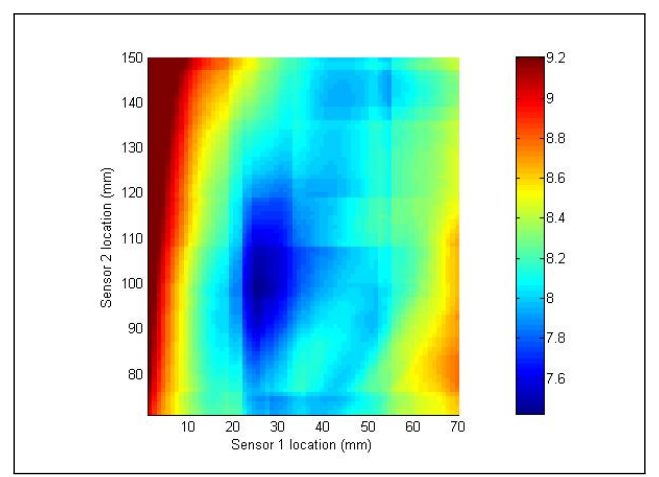

Fig. 3. Cumulated squared tip deflection error of reconstruction on our set of needles depending on differents sensors locations (logarithmic scale).

Fig.3 shows the cumulated tip deflection error from the reconstruction of our set of needles with two sensors. The optimal sensors position is the one for which the minimum reconstruction error is obtained. We see that for two sensors the reconstruction error function is smooth and almost convex.

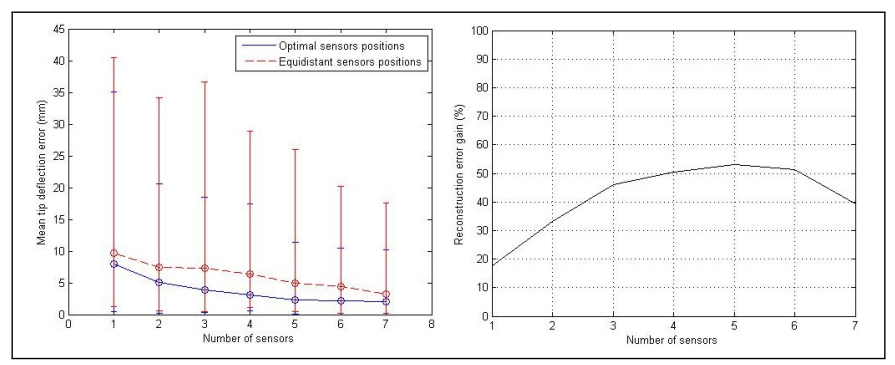

Fig. 4. Left: Tip deflection error for equidistant and optimal sensors positions depending to the number of sensors. Right: Percentage of precision gained by using optimal sensors positions instead of equidistant sensors position

Fig. 4 compares the mean tip error of reconstruction of our set with optimal sensors placement sensors versus equidistant sensors placement. It indicates also the percentage of precision gain by using needle with optimal sensors placement instead of equidistant sensors placement. We can see that the percentage of precision gained is comprised between $18 \%$ (2 sensors) to $52 \%$ (5 sensors).

Fig. 5 presents an exemple of reconstrusction of a needle of our data set with differents number of sensors and differents placement.

\section{Discussions And ConCLUSION}

Fig.I presents the positions for which the solvers converged towards the same solution for a number of sensors comprised between one and seven. As these sensors positions minimize the reconstruction error of our set of needles we can assert that we found the optimal sensors locations for 


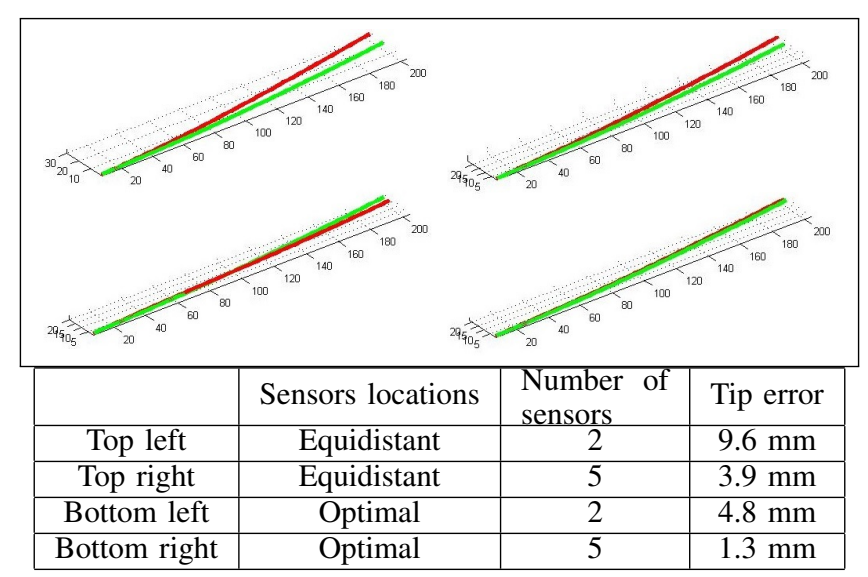

Fig. 5. Comparaison of a needle reconstruction for different number of sensors and differents sensors placement. Shapes in green is the reference needle and shapes in red are reconstruction of the reference needle given data sensors.

this range of number of sensors. The question of optimal locations for a higher number of sensors is not relevant as the number of sensors on a needle is limited. Moreover we see on Fig.4 that the gain between optimal sensor location and equidistant sensor location starts to decrease for a number of sensor superior to five. The reason is that when the number of sensors increase they tend to be placed in an equidistant way.

Fig.4 presents the mean tip deflection error after reconstruction of our reference set of needle. Comparison with other works shows that our mean tip error is correct compared to the length of our needles: for a 4 sensors needle we have a mean tip error of $3.2 \mathrm{~mm}$ for $200 \mathrm{~mm}$ long needles while Abayazid et al. have $1.8 \mathrm{~mm}$ for $120 \mathrm{~mm}$ needles [1]. Here the ratio of mean tip error over the length of the needle is approximately the same. The improvement of the quality of the reconstruction evaluated in the Fig.4 indicates that the reconstruction error falls from $18 \%$ ( 2 sensors) to $52 \%$ (5 sensors) when optimal sensors locations are used compared to equidistant sensors locations. This results exhibits that the gain of precision coming from an optimal sensors placement is not negligible. It confirms also that for a number of sensors going from one to seven our method gives better locations than a standart equidistant placement.

Fig.5 expose the concrete case of one of the needle of our reference set which has a huge deformation (tip deflection superior to $20 \mathrm{~mm}$ ). Thus the reconstruction with only two sensors placed equidistantly gives a tip error of $9.6 \mathrm{~mm}$. Keeping the same number of sensors but with optimized locations divide the tip error by $2(4.8 \mathrm{~mm})$ which is a little bigger than the the tip error with five sensors $(3.9 \mathrm{~mm})$. This example shows here that placing optimally the sensors can be a relevant solution to increase accuracy and should be considered before adding new sensors.

The use of real needle data combined with three dimensional processing contributes to the relevancy of our method by using less restrictive hypothesis. We believe that these choices improved the accuracy of reconstruction and the reliability of the sensors positions results.

\section{REFERENCES}

[1] M. Abayazid, M. Kemp, and S. Misra. 3d flexible needle steering in soft-tissue phantoms using fiber bragg grating sensors. In Robotics and Automation (ICRA), 2013 IEEE International Conference on, pages 5843-5849, May 2013.

[2] Richard L. Bishop. There is more than one way to frame a curve. The American Mathematical Monthly, 82(3):246-251, 1975.

[3] L. Carrat and S. LAVALLEE. Device and method for determining the position of an instrument in relation to medical images, October 11 2012. US Patent App. 13/441,736.

[4] Carl de Boor. A Practical Guide to Splines. 1978.

[5] Rida T. Farouki and Chang Yong Han. Rational approximation schemes for rotation-minimizing frames on pythagorean-hodograph curves. Computer Aided Geometric Design, 20(7):435 - 454, 2003.

[6] C. Fouard, A. Deram, Y. Keraval, and E. Promayon. Camitk: a modular framework integrating visualization, image processing and biomechanical modeling. In Y. Payan, editor, Soft Tissue Biomechanical Modeling for Computer Assisted Surgery, pages 323-354. 2012.

[7] Graham Hocking, Simon Hebard, and Christopher H Mitchell. A review of the benefits and pitfalls of phantoms in ultrasound-guided regional anesthesia. Regional anesthesia and pain medicine, 36(2):162170, 2011.

[8] Sébastien Le Digabel. Algorithm 909: Nomad: Nonlinear optimization with the mads algorithm. ACM Trans. Math. Softw., 37(4):44:1-44:15, February 2011.

[9] A. Leroy, M. Baumann, P. Mozer, J. Troccaz, and V. Daanen. System and method for imaging and locating punctures under prostatic echography, February 5 2013. US Patent 8,369,592.

[10] MATLAB and Global Optimization Toolbox. Release R2014a. The MathWorks, Inc., Natick, Massachusetts, United States.

[11] Yong-Lae Park, S. Elayaperumal, B. Daniel, Seok Chang Ryu, Mihye Shin, J. Savall, R.J. Black, B. Moslehi, and M.R. Cutkosky. Realtime estimation of 3-d needle shape and deflection for mri-guided interventions. Mechatronics, IEEE/ASME Transactions on, 15(6):906915, Dec 2010.

[12] T. K. Podder, D. P. Clark, and J. Sherman. Effects of tip geometry of surgical needle an assessment of force and deflection. 2005.

[13] Christian H. Reinsch. Smoothing by spline functions. Numerische Mathematik, 1967.

[14] Adeline L.G. Robert, Grégory Chagnon, Ivan Bricault, Philippe Cinquin, and Alexandre Moreau-Gaudry. A generic three-dimensional static force distribution basis for a medical needle inserted into soft tissue. Journal of the Mechanical Behavior of Biomedical Materials, 28:156 - 170, 2013.

[15] R.J. Roesthuis, M. Kemp, J.J. van den Dobbelsteen, and S. Misra. Three-dimensional needle shape reconstruction using an array of fiber bragg grating sensors. Mechatronics, IEEE/ASME Transactions on, 19(4):1115-1126, Aug 2014.

[16] Gang Wan, Zhouping Wei, Lori Gardi, Donal B. Downey, and Aaron Fenster. Brachytherapy needle deflection evaluation and correction. Medical Physics, 32(4):902-909, 2005.

[17] Wenping Wang, Bert Jüttler, Dayue Zheng, and Yang Liu. Computation of rotation minimizing frames. ACM Transactions on Graphics (TOG), 27(1):2, 2008.

[18] Wenbin Yang. Conception et intgration de microsystmes sur un cylindre pour la mesure de ses dformations : application un outil du domaine de la sant. PhD thesis, EEATS, 2011.

[19] Wenbin Yang, A. Bonvilain, T. Alonso, A. Moreau-Gaudry, and S. Basrour. Modelling and characterization of an instrumented medical needle in sight of new microsensor design for its insertion guidance. In Engineering in Medicine and Biology Society (EMBC), 2010 Annual International Conference of the IEEE, pages 6465-6468, Aug 2010.

[20] Jincong Yi, Xiaojin Zhu, Hesheng Zhang, Linyong Shen, and Xiaoping Qiao. Spatial shape reconstruction using orthogonal fiber bragg grating sensor array. Mechatronics, 22(6):679 - 687, 2012. Special Issue on Intelligent Mechatronics (LSMS2010 \&amp; ICSEE2010). 